\title{
Determinación y causalidad en salud colectiva. Algunas consideraciones en torno a sus fundamentos epistemológicos
}

\author{
Determination and causality in Public Health. \\ Some considerations on its epistemological foundations
}

Juan M anuel M endoza Rodríguez ${ }^{1}$

Edgar Carlos]arillo Soto ${ }^{1}$

${ }^{1}$ Ciencias en Salud Colectiva, Universidad Autónoma M etropolitanaXochimilco. M éxico, D.F. manolo_97@yahoo.com
Abstract The discussion on the determinants is extremely prolific in the area of health. However, we still have to think about the way in which the determination isdefined and understood as a category that allows to question reality, from an overall and complex view, and the extent that they can have their application in public health. This article discusses the concepts of causality, which is dominant in the positivist vision as well as determination and historicity, used by some alternative views on health. Some arguments are exposed on the ontological and epistemological approach to address the study of health-disease process, from considerations of phenomenology. Key words Determination, Cause, Objectivity, Historicity, Happening, Be-there
Resumo La discusión sobre los determinantes es muy prolífica en el campo dela salud, no obstante todavía hace falta reflexionar sobre la manera en queésta es definida y entendida como una categoría que permite problematizar la realidad desde una mirada totalizadora y compleja y sobre los alcancesque puede tener su aplicación en la salud colectiva. Estetrabajo discutelos conceptos decausalidad, que predomina en la visión positivista, y determinación utilizado por algunas visiones alternativas en el campo de la salud. Se exponen algunos argumentos en torno al tratamiento ontológico y epistemológico en el estudio del proceso salud-enfermedad a partir de algunos planteamientos de la fenomenología.

Palabras clave Determinación, Causa, Objetividad, Historicidad, Acontecimiento, Ser-ahí 


\section{Introducción}

La salud colectiva se ha desarrollado como campo deconocimiento y acción con iniciativas para la transformación de la realidad social, desde donde se problematiza y construye el objeto de estudio demanera transdisciplinaria, totalizadora y compleja $a^{1-4}$. Es un campo innovador del conocimiento en el quese debereflexionarsesobrelos conceptos que le dan sustento. La reflexión es necesaria, como apunta Ayres ${ }^{5}$, para la edificación de una postura crítica "consistentey madura" a partir de bases sólidas para impulsar el desarrollo de un pensamiento a través del cual sea posible comprender y transformar la realidad como un todo complejo y dinámico.

Aunque tanto la salud colectiva como la salud pública centran su atención en los problemas relacionados con la salud de los grupos humanos, existen diferencias fundamentales que permiten delimitar claramente cada campo. Una de las diferencias más críticas se relaciona con la manera en la queseentiendey explica la sucesión de fenómenos involucrados en el proceso saludenfermedad. El concepto de determinación, en contraposición ala noción decausa, permitepensar el objeto de estudio dela salud colectiva como algo distinto al objeto de la salud pública. En lugar del determinismo causal basado en la triada agente-huésped-ambiente utilizado comúnmente por la salud pública, la salud colectiva entiende el proceso salud-enfermedad desde la totalidad constituida por las formas específicas en las que la sociedad se organiza y se reproduce política, económica y culturalmente ${ }^{6}$. Deestaforma el carácter social e histórico de la salud y la enfermedad puede explicarse asumiendo queson momentos deun mismo proceso, quesi bien tiene quever con la plasticidad del organismo para responder "a través de sus condiciones de desarrollo"7, también ocurre en un contexto de existencia de los individuos en la sociedad y en un momento del devenir histórico.

Sin embargo, en el campo de la salud losconceptos de causalidad y determinación presentan dificultades al momento de definirlos, ya que no existe una única interpretación acerca de ellos. Por ello, es necesario reflexionar sobre su naturaleza y definir los al cances queel análisis crítico de los mismos puede tener en la comprensión de la salud y la enfermedad. En el presente trabajo se plantea una perspectiva en torno a estos supuestos básicos que han definido el pensamiento y la práctica dealgunas delas visiones en el campo de la salud. Particularmente, se analizan algunos argumentos que permiten definir los supuestos epistemológi cos desde la salud pública, la salud colectiva y la filosofía, así como el aporte que puededar la visión fenomenológica dela causalidad y la determinación al estudio de la salud.

La causalidad en el modelo epidemiológico

La causalidad según Hume, puede ser entendida de dos maneras: (1) de un objeto A se sigue uno $B, y(2)$ si un primer objeto no tiene lugar, un segundo no puede existir ${ }^{8}$. En el primer caso, la causalidad establece una relación necesaria y unívoca entre fenómenos. Es necesaria porque la ocurrencia deun fenómeno sólo puede debersea la presencia de al go que le antecede, y es unívoca porque lo que antecede dará lugar a su consecuente y sólo a ese consecuente. En la clásica noción de la historia natural dela enfermedad, todo el modelo explicativo está fundado en este supuesto. En el segundo caso, la causalidad denota regularidades accidentales desucesión, y por tanto suficiencia o probabilidad ${ }^{9}$ que, como en el modelo de Mc Mahon y Pugh ${ }^{10}$, es un fundamento central de muchos análisis estadísticos característicos de la epidemiología.

La causalidad probabilística puede explicarse a través de relaciones condicionales, según la necesidad y la suficiencia de los el ementos queestán presentes en la relación. De esta manera, dado que a la causa no siempre le sigue un efecto, se pueden establecer distintos tipos de relaciones:

(1) Relación necesaria y suficiente: Si A entonces $B$, y si B entonces A.

(2) Relación necesaria y no suficiente: Si A entonces $B$ o no $B$, y si $B$ entonces $A$.

(3) Relación no necesaria y suficiente: Si A entonces $B$, y si $B$ entonces $A$ o no $A$.

(4) Relación no necesaria y no suficiente: Si A entonces $B$ o no $B$, y si B entonces $A$ o no $A$.

Por ejemplo, el modelo epidemiológico de Rothman ${ }^{11}$ utiliza, para establecer el riesgo de enfermar de una población, relaciones causales delos tipos 1, 2 y 3 . El efecto observado se da por la presencia de causas necesarias o suficientes, aunque las causas suficientes pueden constituirse a partir de un conglomerado de causas componentes. Así, las causas componentes son relaciones del tipo 4, pero sólo pueden producir un efecto si conforman una causa suficiente (tipos 1 y 3). En este sentido la enfermedad se presenta cuando hay una causa suficiente, pero ello implica que debe haber una causa necesaria, por lo tanto, la causalidad se reduce a relaciones de los 
tipos 1 y 2. Deesta manera, el principio básico de la causalidad: A entonces $B$, se mantiene; lo que cambia son las condiciones bajo las cuales este principio puedeser aceptado en la explicación de un fenómeno. Tales son los criterios de Bradford $\mathrm{H} \mathrm{ill}^{12}$, utilizados para establecer inferencias causales en epidemiología.

De manera general, la salud pública asumela causalidad como un principio que existe en sí mismo y que puede ser observado en la naturaleza, que regula toda sucesión de fenómenos y a través del cual pueden establecerse relaciones verificables y predecibles. Esta postura asume que los fenómenos son "condiciones objetivas", entendiendo "condición objetiva" como existencia propia e independiente de cualquier interpretación. Es decir, que solo podemos conocer a través de los sentidos. Pero si sólo podemos conocer los quees puesto antela vista y la razón es incapaz de encontrar en la diversidad de sus formas aquello que los une, es decir, de realizar una síntesis, no seríamos tampoco capaces de formular reglas que puedan ser aplicadas a fenómenos. La matemática y la física, por ejemplo, no encontrarían un sustento en el cual apoyar la necesidad de sus proposiciones.

Desde el punto de vista de la fenomenología, un objeto no es lo que está al alcance de nuestros sentidos. Los sentidos se limitan a la percepción de fenómenos, representaciones que tenemos de los objetos. Para poder conocer el objeto hay que hacer uso de la razón, pensar el fenómeno para poder extraer su esencia: el objeto. En este sentido se dicequela objetividad de un conocimiento, no proviene de la experiencia, ya que a través de ella lo único que podemos captar son fenómenos. Por ello, no es posible derivar de la experiencia el carácter objetivo de la causalidad.

\section{La causalidad como representación a priori}

Según Kant el carácter objetivo de la causalidad sólo puede ser explicado a priori, debido a que el concepto de causa se funda en el entendimiento porque, que $A$ siga a $B$ es una representación mental que hace posible conocer al go como objeto, donde la sucesión no es más que una representación de tiempo que nos permite organizar los fenómenos ${ }^{13}$. Una representación a priori implica ideas, conceptos, juicios o raciociniosque no surgen de la experiencia, sino que se han producido a partir de razonamientos. No obstante, las representacionesa priori deben poder ser aplicadas a objetos de la experiencia, porque de otro modo no tienen ninguna utilidad. Lo que aquí Kant está planteando es muy importante, ya que la lectura poco atenta a este pasaje ha dado lugar a serias confusiones. No hay una preeminencia de la razón sobre los sentidos o de los sentidos sobre la razón, lo que el autor critica es precisamente la valoración absoluta de una o la otra.

La causalidad como representación a priori es necesaria y universal; necesaria porque si tal concepto no estuviera formado en nuestro entendimiento antes detener al guna experiencia no podríamos conocer, y universal porque se aplica a todos los fenómenos de la experiencia. Sin embargo, aquí no se está hablando de la necesidad en el mismo sentido en que se expuso cuando se hablaba del modelo condicional. Hay que tener claro que el carácter necesario de la causalidad no se refierea que una relación causal sea necesaria, sino a que el esquema general de la causalidad debe estar en nuestro entendimiento para poder establecer queuna relación entrefenómenos es una relación de tipo causal.

Por tanto, si el principio de causalidad no deriva de la experiencia, sino de la razón, es decir, es una determinación a priori, entonces no es posible establecer, a través de la medición de la sucesión de fenómenos en el tiempo, la causa de la enfermedad. Lo que sí puede hacerse, es determinar apriorísticamente la relación entre fenómenos: aplicar un concepto abstracto (causalidad) a objetos de la experiencia. Al hacer esto, el valor objetivo del conocimiento obtenido a partir deestablecer esta relación no yaceen su carácter empírico, sino en la acción subjetiva del sujeto que conoce. En este caso, "determinar", debe entenderse desde el punto de vista fenomenológico, es decir, agregar un predicado real a un concepto para ampliar su conocimiento ${ }^{13}$. La determinación en este sentido no es una determinación real, sino lógica. De esta manera, hay determinaciones que tienen su origen en la experiencia, y determinaciones quetienen su origen en la razón. Por su carácter apriorístico, la causalidad es una determinación lógica y no una determinación empírica.

Desde cierta perspectiva, el determinismo se ha entendido como una relación causal necesaria y unívoca ${ }^{14}$. Sin embargo, estas características de la causalidad, no son las quela hacen ser determinante. Si bien la causalidad es una determinación, no toda determinación es causalidad, por tanto, no se puede decir, desdeun punto de vista ontológico, que casualidad es igual a determinación ${ }^{15}$, entendida como una relación total y absoluta entre una causa y su efecto. La tendencia a confundir o reducir la determinación a la causalidad es 
un problemaal queseenfrenta la formación científica ${ }^{16}$ que ha estado presente desde etapas muy antiguas del conocimiento ${ }^{17}$, y queen el campo de la salud hasido columna vertebral del pensamiento explicativo de su objeto de estudio.

\section{La categoría determinación en salud colectiva}

El interés de la salud colectiva por definir la determinación y de ubicarla como categoría explicativa de los procesos sociales en torno a la salud-enfermedad-atención-muerte, surge de la necesidad de superar la visión reduccionista sobre el origen de las enfermedades, la cual se caracteriza por una perspectiva donde la realidad compleja se limita a expresiones sistémicas y el supuesto empirista que niega la posibilidad de conocer la esencia de las $\operatorname{cosas}^{18}$.

En la salud colectiva la determinación es una categoría central en la explicación del proceso salud-enfermedad. El planteamiento básico es que, en última instancia, las condiciones materiales de existencia determinan la distribución desigual de la salud-enfermedad en los grupos humanos. El término "determinantes de la salud" es utilizado por primera vez por Thomas McKeown ${ }^{19}$ para explicar la disminución de la mortalidad infantil en Inglaterra y Gales durante el siglo XIX. En un trabajo posterior, M cKeown ${ }^{20}$ dice que las condiciones sociales son las causas fundamental es de la enfermedad, ya que el mejoramiento de la salud que experimentó la sociedad durante este período, se debe más a las mejoras en las condiciones económicas que a los avances en medicina o las intervenciones en salud pública.

La salud colectiva atiendeal proceso colectivo en el que se producen y reproducen las condiciones sociales que llevan a enfermar de una manera específica a los grupos humanos, según su inserción en el proceso de producción. Desde la salud colectiva, los patrones de producción y consumo son considerados determinantes fundamentales del proceso salud-enfermedad, pues es a partir del estudio de las contradicciones entrevalores de uso y fuerzas deteriorantes, generadas por el modo en que se organizan y reproducen socialmentelos gruposhumanos, quesepueden entender los problemas de salud-enfermedad ${ }^{21}$.

Al concebir la salud-enfermedad como un proceso colectivo de producción y reproducción social, la salud colectiva argumenta que este proceso está determinado por el desarrollo de las fuerzas productivas, las relaciones de producción y la ideología del grupo dominante, porque son estas formas específicas con las que el hombre entra en contacto con la natural eza y la transforma socialmente. Es decir, que la forma en la que el humano se apropia dela naturaleza está determinada por el modo de producción en una formación social determinada, pero además por cómo se articulan entre sí distintos modos de producción o formaciones sociales en un momento histórico específico ${ }^{22}$. A su vez, el modo de producción implica un cierto desarrollo de las fuerzas productivas y ciertas relaciones de producción e intercambio, que determinan la frecuenciay distribución dela salud-enfermedad en Ios colectivos. Lo que los hombres hacen determina la salud-enfermedad como fenómeno material, que todavía no es pensado por el hombre; la forma en que es caracterizado el fenómeno material defineel proceso salud-enfermedad. Este carácter doble: ideológico y material del fenómeno biológico individual es lo quele da su carácter de determinación social.

El proceso salud-enfermedad adquiere historicidad por que los procesos donde tiene lugar son circunstancias delosindividuos quehan construido en el devenir y cadauna deesas circunstancias está definida en condiciones y contextos específicos dela sociedad; es decir, queno se puededar cuenta de ello al margen del momento histórico en el que se desarrolla ${ }^{18}$. Esta es una premisa que ha definido la mirada de la medicina social y la salud colectiva. En este campo de conocimiento los mayores aportes en torno al concepto de determinación se han dado desdeel plano metodológico y epistemológico; no obstante, es necesario volver a plantear la pregunta, no sólo desde estos planos de análisis, sino también y primariamente, desdeel plano ontológico.

En una discusión sobre los distintos usos que selehan dado al concepto determinación, Breilh ${ }^{23}$ apunta tres definiciones que son utilizadas frecuentemente en el campo científico. La primera es la determinación como características definidas de un proceso, la segunda tiene que ver con relaciones de univocidad y la tercera con modos de devenir. Esta última acepción, nos dice el autor, explica mejor la génesis de los procesos sin llevarnos hacia el determinismo. Esto nos conduce a la fenomenología, y entonces determinación no tienequever con la medición de relaciones, ya necesarias, ya probabilísticas; sino con la condición subjetiva denuestra humanidad ¿Pero de qué manera puede ser aprendido su carácter histórico en su totalidad y complejidad? Si entender la determinación como modo de devenir nos 
guía hacia la comprensión de la génesis de los procesos, se debe decir entonces que la historicidad del proceso salud-enfermedad no se explica únicamente por la presencia de ciertas enfermedades a lo largo deun periodo. Un acontecimiento histórico no es al go que ha quedado atrás en el tiempo, sino que adquiere permanencia en y a través de la actividad humana, y es esta misma permanencia lo que hace que se continúe. Así, la enfermedad como determinación, no es sólo un acontecimiento queocurreen el tiempo, sino que el tiempo trascurrido del sujeto en su entorno de producción y reproducción social es el queexplica la presencia de la enfermedad.

Tanto en la salud pública como en la salud colectiva, el tiempo se concibe como una línea quese puedeextender indefinidamentey queexistencia en sí mismo; en la fenomenología, el tiempo no existe propiamente, el tiempo es algo que constituye la esencia del humano, y lo que hace posiblecualquier determinación.

\section{La determinación como representación} a priori en el estudio dela salud enfermedad como proceso socio-histórico

Las nociones de Tiempo, Ser y Mundo, representan en la filosofía de Kant conceptos trascendentales, porque no podemos utilizar otros conceptos de mayor generalidad para explicarlos, sino que estos son los conceptos más generales que existen. Ellos, pueden ser comparados con Historicidad, Sujeto y Espacio, que designan categorías de un alto grado de abstracción en la salud colectiva.

El humano, está representado en la filosofía de Heidegger por el "ser-ahí. "Ser-ahí" implica "ser-en", es decir queno solo es en sí mismo, sino que es en algo y ese algo es el mundo ${ }^{23}$. Por ello, "ser-ahí" hace referencia a estar-siendo-en-elmundo. Pero el mundo no es sólo lo que está "ahí afuera", sino que el mundo es también "ahí dentro". El mundo no es independiente denosotros, es parte de nosotros. El ser-ahí determina los objetos de su mundo circundante cuando se detiene a mirarlos y es capaz de comprenderlosy formar una proposición sobre ellos. Por eso es un ser determinante, pero también determinado, porque se relaciona con otros "ser-ahí" que también están en el mundo.

Considerando que desde una perspectiva ontológica, determinación es la manifestación de ser del humano en el mundo - en la que se agregan significados a los objetos de la experien- cia, permitiendo ampliar el conocimiento quetenemos de ellos -, y que los modos de ser posibles son siempre modos temporarios, se puede establecer una explicación fenomenológica. Desdela perspectiva de $M$ artin $\mathrm{H}$ eidegger se pueden retomar las categorías de la salud colectiva en los siguientes términos: determinación es la manifestación de la subjetividad del sujeto que se desenvuelve en el espacio social, dando significado y sentido a los objetos, acciones y relaciones que establece en ese espacio. Lo que sirve de fundamento a la acción subjetiva, es decir lo que no cambia y que es la esencia del sujeto, es su carácter temporal; el sujeto no puedeliberarse del tiempo como base a través de la cual interpreta todo lo que capta mediante los sentidos. En efecto, la noción que tenemos del tiempo sirve para interpretar el movimiento de las cosas, las acciones individuales y colectivas y las relaciones; sirve, pues, para comprender, como resultado de un proceso de interacción social, el fenómeno biológico al que denominamos salud-enfermedad.

Determinar, es el decir del sujeto sobre los objetos de la experiencia; pero el decir del sujeto está siemprefundado en su carácter deser-en-elmundo, o sea, en la posición que guarda en el espacio social; pero este decir también se funda en su propio carácter temporal, porque interpreta el mundo siempre desde su posición temporal/finita, es decir, en un momento histórico en el que están dadas de cierta forma las características del espacio social, donde se enfrenta a ciertas condiciones que le permiten relacionarse con cosas y sujetos de una manera específica.

En la fenomenología de Heidegger, determinación ehistoricidad son conceptos que permiten comprender al "ser-ahí" en su forma de ser cotidiana, como un ser que conoce no sólo interiorizando los objetos de la experiencia, sino también expresando en los objetos su subjetividad ${ }^{24,25}$. Por ello, la historicidad del "ser-ahí" no se comprende colocando el ser en unalínea detiempo, sino comprendiéndolo como origen de la temporalidad. No podemos concebir la historia como "fuera de nosotros", porque todo acontecimiento es posibleen tanto relación de representaciones determinada por nuestra conciencia. El cambio no es un carácter del tiempo en sí, sino de los fenómenos en el tiempo, y como éste no puede ser percibido en sí mismo, se debe buscar en sus determinaciones (los fenómenos) lo que no cambia y que es el substratum de toda simultaneidad y sucesión.

En esa perspectiva, lo que hay que entender es que con "determinación" se significa algo distinto a lo que comúnmente se representa en la 
concepción empirista de causa, se quiere hacer patente "al go más" allá de la experiencia que nos muestra el puro poner "ante los ojos juntas" cosas que se suceden. Se trata de abandonar la preeminencia de la sustancia sobre la existencia y de reconocer como sustancia la propia existencia. De otro modo el argumento cartesiano sustancialista triunfa y todo el conocimiento estaría dado por la razón, y no tendría ningún fundamento empírico. Por el contrario, si pensamos la determinación como la relación necesaria entre fenómenos, cualquiera que sea la naturaleza de estos, cualquier reflexión, cualquier intento de ir más allá de lo que "aparece ante los ojos", será vacío. Así, la única posibilidad de salvar el sustancialismo, puede decirse cualquier especie de trascendentalismo sin negar el conocimiento de las sustancias, es preguntar por la existencia. Esto debe ser necesariamente interrogar por "quién" y no por "qué". La pregunta planteada así abre la posibilidad de acceder al ser en su esencia; es decir en su modo de ser histórico. De esta manera, sepuede conocer la historicidad del ser si secomprende el "ser-ahí" como un ser determinante.

Entonces, comprender desdeun punto devista histórico comienza en ocuparnos de los enunciados sobreciertos acontecimientos que ocupan nuestro interés, detenernos ante proposiciones que los sujetos construyen en el "decir algo" y avanzar después haciala comprensión deeso que sedice. Aquí es dondeel interpretar fenomenológico nos permite el acceso a aquello que la palabra guarda en su decir. Sin embargo, como el lenguaje no es el lugar primario de la verdad y no toda interpretación nos lleva a la verdad ${ }^{24}$, sedebe ir hacia la articulación de una fenomenología hermenéutica26,27 para la salud colectiva que nos permita pensar dialécticamentelas prácticas concretas que los sujetos individuales y colectivos realizan ante situaciones de salud-enfermedad y en su específica manera de insertarse en el movimiento general de la reproducción social.

Esta articulación fenomenológico-hermenéutica pude plantearse a partir estudiar los diversos discursos sobre la salud-enfermedad, y buscar en ellos su esencia a través deinterpretarlos. Pero ¿cuáles son las maneras de interpretación que podemos considerar válidas? ¿Cuáles son los límites que pueden ser fijados sin caer en un proceso interpretativo quenos lleveindefinidamente hacia cualquier parte? 0 por el contrario, y esta la cuestión que aquí nos interesa porque en ella se da el interrogar ontológico, ¿cuál es el modo de interpretar que nos lleve más allá del "decir al go" y sea capaz de develar el "al go" en el "decir"?
H eidegger ${ }^{24}$, al colocar el lenguaje como una forma en la que el "ser-ahí" puede ser comprendido históricamente ${ }^{26}$, abre esta posibilidad. A través del lenguaje, la esencia del ser se manifiesta, pero para poder acceder a ella, hay que hacer las preguntas pertinentes, y esas preguntas deben estar dirigidas al ser.

Implicaciones para la práctica y la investigación en la salud colectiva

Como sehavisto, la determinación, desdeun punto de vista fenomenológico, no se deriva de la sucesión temporal lineal, como en: A luego B. El tiempo no es una línea que se prolonga al infinito, nosotros lo representamos así, porque es la manera en que podemos comprender la sucesión o simultaneidad delas relaciones defenómenos. En sí mismo, el tiempo no tieneninguna figura, ninguna forma; sin embargo, necesitamos dotar a esta representación de una forma que pueda ser aplicada a los objetos de la experiencia.

Dado entonces, quela representación del tiempo no puedederivarse dela experiencia, sino que más bien, puedeser aplicada a la experiencia, tiene un carácter a priori. En ese sentido, es que se entiende el tiempo en la fenomenología-hermenéutica. La importancia que tiene esta concepción del tiempo, es que, en primera instancia, la objetividad del conocimiento no está ya dada en la observación de hechos que conocemos tal y como se dan a nuestros sentidos, sino en el carácter a priori de los conceptos, siendo estos conceptos los que hacen posible la observación empírica. Si no se tiene en el intelecto la noción de causa anterior a una experiencia, no es posible establecer reglas de causalidad, por ejemplo, que de $A$ sigue $B$, y es por ello necesaria.

Una segunda implicación deesteconcepto de tiempo es que permitedarleun papel fundamental al sujeto, el "ser-ahí"; porque el tiempo es la esencia original del humano, no algo independiente de él. El humano es temporal, no sólo por su finitud, sino porque el tiempo se gesta en él y al mismo tiempo lo constituye. Así, preguntar por la relación de los fenómenos en el tiempo es preguntar por la manera en que el humano se manifiesta, pero también cómo es afectado por su forma de ser y la forma de ser del otro con el que se enfrenta al estar siendo.

La determinación supone una organización de fenómenos que se suceden en el tiempo, entendido como la dimensión de existencia del humano, la esencia del ser-ahí. Por lo tanto la sa- 
lud-enfermedad devela la propia existencia del sujeto y su mirada de sí y de los otros, señala la dimensión de su existencia en un aquí y ahora. No se trata solamente deuna ordenación de procesos en el tiempo, de nexos y relaciones entre fenómenos. Tampoco de la causalidad que surge de la experiencia y por lo mismo de la causalidad a posteriori.

La determinación es una representación a priori, lógica, organizadora de la complejidad y de la totalidad. Es un poner en perspectiva dimensiones y procesos que permiten el entendimiento de la existencia del ser como universalidad y como concreción que conserva la esencia delo quehacambiado y al mismo tiempo el cambio de lo que permanece. Es así, una forma de comprender la salud-enfermedad como producto de la propia existencia del humano, pero no el humano aislado, en su pura individualidad, sino como productor y partícipe de la propia acción transformadora de la sociedad en que se desenvuelve; y por lo tanto, como génesis y consecuencia de la enfermedad, de la salud, del bienestar.

Entender de esta manera la determinación, permiteir más allá de establecer relaciones lineales entrefenómenos, dedescribir la pura forma y descubrir la esencia de los fenómenos; nos habla de sujetos y no de objetos, de relaciones complejas producidas y reproducidas en el seno de la sociedad donde estos sujetos interaccionan y generan sus condiciones de existencia.

La noción de complejidad donde los sujetos sedesenvuelven, desdela mirada ontológica dela realidad recortable para su análisis o la imposibilidad de abarcamiento del todo, representa en los hechos, un posicionamiento que lleva a interpretar la causa como eventos aislados del sujeto, su devenir y sus nexos relacionales. En cambio, a través del pensamiento fenomenológico se puede ir más allá de los límites de la experiencia, es decir hacia lo trascendental, sin caer en el terreno de lo aparente al que nos puede conducir el uso absolutamente a priori de los conceptos.

Para la salud colectiva, que busca ir más allá de la pura forma que nos muestra la experiencia sensible y pretende descubrir lo que hay de sustancial en el fenómeno, este modo de preguntar existenciario aporta elementos que pueden servir al pensamiento crítico en el estudio del proceso salud-enfermedad, porque al mostrarse la apariencia, 0 en términos de Kosik ${ }^{28}$, la pseudoconcreción, se hace posible su destrucción. De esta manera la historicidad se hace patente cuando logramosver en la actividad trascendente denuestra conciencia sus determinaciones. La historicidad, concebida así, nos permite preguntar por el sistema de relaciones entrefenómenos como una manifestación de nuestra subjetividad, es decir, conocer la manera en la que los objetos de la sensibilidad nos afectan, y no cómo se relacionan necesariamente y con absoluta universalidad. Hace posible, en fin, un modo de apertura, y no un rígido modo de pensar que si bien, nos asegura al gunas certezas, cierra al mismo tiempo el acceso a aquello por lo que son posibles todos los objetos de conocimiento.

\section{Colaboradores}

JM M Rodríguez fue responsable por la concepción y la redacción final. ECJ Soto fue responsable por la revisión crítica y la redacción final. 


\section{Referencias}

1. Breilh J. Nuevos Conceptos y Técnicas de Investigación. Guía pedagógica para un taller de metodología. Ecuador: CEAS; 1994.

2. Jairnilson P, Almeida N. Saúde Coletiva: Uma nova saúde pública ou campo aberto a novos paradigmas? Rev. Saude Publica 1998; 32(4):299-316.

3. Almeida N, Silva J. La crisis de la salud pública y el movimiento de la salud colectiva en Latinoamérica. Cuadernos M édicosociales 1999; 75:3-30.

4. Granda E. ¿A qué Llamamos Salud Colectiva, Hoy? Rev Cubana Salud Púbica 2004; 30(2):148-159.

5. Ayres J. Conceptos y prácticas en salud pública: algunas reflexiones. Rev Fac N ac Salud Pública 2002; 20(2):67-82.

6. Laurell AC. Sobre la concepción biológica y social del proceso salud-enfermedad. En: Rodríguez MA, compilador. Lo biológico y lo social. Serie Desarrollo de Recursos Humanos no 101. Washington, D.C.: OPS/OM S; 1994. p. 1-19.

7. Breilh J. La salud-enfermedad como hecho social. En: Deterioro de la vida en Ecuador. Quito: CEAS 1989.

8. Lewis D. Causation. J Phil. 1973; 70(17):556-567.

9. Rosen D. In defense of a Probabilistic Theory of Causality. Phil Sci. 1978; 45(4):604-603.

10. MC Mahon B, Pugh T. Principios y métodos de epidemiología. M éxico: La Prensa M édica M exicana; 1981.

11. Rothman K. Epidemiología moderna. México: Ediciones Díaz Santos; 1973.

12. Hill AB. The environment and disease: association or causation? Proc R Soc M ed. 1965; 58:295-300.

13. Kant I. Crítica de la Razón Pura. Tomo I. M éxico: Colofón; 1996.

14. Carr EH. ¿Qué es la Historia? España: Ariel; 2004.

15. Anscombe E. Under a Description. Noûs 1979; 13(2):219-233.

16. Schield M. Correlation, Determination and Causality in Introductory Statistics. American Statistical Association, Section on Statistical Education. 1995. [cited 2008 Feb 10]. Available from: http://web. augsburg.edu/ schield/M iloPapers/95ASA.pdf
17. Bunge M . La causalidad: el principio de causalidad en la ciencia moderna. Buenos Aires: Editorial Universitaria de Buenos Aires; 1972.

18. Laurell AC. La salud-enfermedad como proceso social. Rev Latinam Salud. 1981; 2(1):7-25.

19. McKeown T. An interpretation of the modern rise in population in Europe. Population Studies. 1972; 26:345-382.

20. McKeown T. El papel de la medicina: ¿sueño, espe jismo o némesis? M éxico: Siglo XXI; 1982.

21. Breilh J. Epidemiología: economía, medicina y política. Quito: Universidad Central; 1979.

22. Laurell AC. Investigación en sociología médica. Salud Problema 1978; 2:18-20.

23. Breilh J. Epidemiología Crítica. Ciencia emancipatoria e interculturalidad. Buenos Aires: Lugar Editorial; 2003.

24. Heidegger M. Ser y Tiempo. M éxico: FCE; 2004.

25. Heidegger M. La tesis de Kant sobre el ser. En: ¿Qué es metafísica? y otros ensayos. Buenos Aires: Fausto; 1992.

26. Hermann F. La segunda mitad de Ser y Tiempo. Sobre los problemas Rogers K. Hume on necessary causal connections. Philosophy 1991; 66(258):517-521.

27. Gadamer HG. Verdad y M étodo. Salamanca: Ediciones Sígueme; 1991.

28. Kosik K. Dialéctica de lo concreto. M éxico: Grijalbo; 1975.

Artigo apresentado em 16/06/2008

Aprovado em 10/10/2008

Versão final apresentada em 12/11/2008 